(In: Am. J. Phys. 53 (11), November 1985)

\title{
Common sense concepts about motion
}

\author{
Ibrahim Abou Halloun a) and David Hestenes \\ Department of Physics, Arizona State University, Tempe, Arizona 85287
}

Common sense beliefs of college students about motion and its causes are surveyed and analyzed. A taxonomy of common sense concepts which conflict with Newtonian theory is developed as a guide to instruction.

\section{INTRODUCTION}

In the preceding article, ${ }^{1}$ we established a need for physics instruction which takes the initial common sense (CS) beliefs of students into account. Other investigators ${ }^{2-9}$ have identified specific CS beliefs that conflict with Newtonian theory and so interfere with physics instruction. But a more systematic and complete taxonomy of CS beliefs is needed for efficient instructional design. The purpose of this article is to survey and categorize cs concepts of motion which should be taken into account in mechanics instruction. We are aiming for a comprehensive picture of CS concepts which includes the insights of previous investigators as well as some observations of our own.

In this article we will not attack the difficult problem of designing instruction to accommodate CS preconceptions. But let us note that CS concepts cannot be avoided in physics instruction, for common sense is a codification of experience providing meaning to our natural language. Discourse on physics would be impossible without it. Indeed, physics and science in general can be regarded as an extension and modification of common sense. Conventional physics instruction frequently appeals tacitly to common sense knowledge, but students have trouble when that knowledge is faulty. It is difficult for students to determine exactly what common sense knowledge is reliable without an explicit critique of CS concepts. Our survey of CS concepts of motion is intended to provide a basis for such a critique.

CS beliefs which are incompatible with established scientific theory are quickly labeled as "misconceptions" and dismissed by most scientists. But students are not so easily disabused of CS beliefs, because their own beliefs are grounded in long personal experience. CS misconceptions are not arbitrary or trivial mistakes. Indeed, every one of the misconceptions about motion common among students today was seriously advocated by leading intellectuals in pre-Newtonian times. Historians ${ }^{10-14}$ tell us about the long and difficult critique and analysis of CS beliefs that prepared the way for the "Newtonian revolution." If the evaluation of common sense was so difficult for the intellectual giants from Aristotle to Galileo, we should not be surprised to find that it is a problem for ordinary students today. Accordingly, common sense beliefs should be treated with genuine respect by instructors. They should be regarded as serious alternative hypotheses to be evaluated by scientific procedures. This would provide students with sound reasons for modifying their beliefs beyond the mere authority of teacher and textbook.

Historians have not failed to observe that the great intellectual struggles of the past provide valuable insights into the conceptual difficulties of students. Accordingly, they advocate a strong dose of history for the physics curriculum. I. Bernard Cohen, for example, has written a noteworthy book ${ }^{11}$ on the early history of mechanics for high school students. But the curriculum leaves little room for the history of science, so the topics for study must be chosen judiciously. Topics of great historical interest may be of limited pedagogical value. For example, the rise and fall of the 
Ptolemaic theory is one of the most common historical topics, but it is not concerned with conceptual issues that bother students. On the other hand, Aristotelian misconceptions about free fall are common among students and difficult to rectify.

As a historical background against which to view the cs beliefs of contemporary students, some major ideas of pre-Newtonian physics are reviewed in Secs. II and III. We confess to some oversimplifications of the historical record in our effort to sort out historical facts with pedagogical relevance.

In Sec. IV we report on our own observations of CS beliefs held by college students. They confirm the results of previous investigators, especially about the prevalence of Impetus concepts.

Finally, in sec. V we present a taxonomy of CS beliefs to be used as a guide for instructional design.

\section{ARISTOTELIAN PHYSICS}

Aristotle was the first to systematically develop explicit formulations for cS beliefs about physical phenomena and organize them into a coherent conceptual system. He thus prepared the way for a critique of CS beliefs that contributed to the development of physical science. The long gestation time for science shows how difficult it was to detect and correct the flaws in the Aristotelian system.

The belief systems of students untutored in physics are sometimes characterized as "Aristotelian." The term is inappropriate. Not only is the Aristotelian system far more elaborate and logically consistent, but the belief systems of most students are closer to the medieval Impetus theory, which we discuss later. Many students do hold some Aristotelian beliefs. But for pedagogical purposes, it is advisable to be specific about these beliefs.

Aristotle separated cosmology from the rest of physics, and separate they remained until the "Newtonian synthesis." Aristotelian cosmology is far removed from the thinking of students today, so we can restrict our attention to Aristotle's terrestrial physics. More specifically, we consider only his theory of motion.

Aristotle defined motion as change of position, and he recognized the need for a reference frame with respect to which motion is observed. His arguments that the surface of the earth must be at rest will not occur to the typical student, who believes the earth is rotating only because he has been told. Nevertheless, it may be worthwhile to present the arguments in full force to physics students for them to refute. That would be a significant test of their understanding of Newtonian mechanics. For this purpose, the account of Cohen ${ }^{11}$ may be helpful.

Aristotle regarded motion in a vacuum as an unrealistic abstraction. He supported this opinion with several different arguments. In particular, he argued that motion in a vacuum would be impossible. His arguments do not seem to be of pedagogical interest. The important point is that his theory of motion deals only with bodies immersed in a material medium.

For Aristotle, rest is the "natural state" for all objects, and every motion has a cause. Aristotle recognized two kinds of cause or force: (1) an inherent force or tendency of every object to seek its natural place and (2) a contact force (push or pull) exerted by some external agent (object or medium). He did not admit long-range forces.

The inherent tendency of an object to move toward its natural place depends on its composition. Heavy bodies, composed mainly of earth and water, are endowed with the property of gravity, a centripetal tendency to move toward the center of the universe. Light bodies, composed mainly of air and fire, are endowed with the property of levity, a centrifugal tendency to flee the center of the universe. All this is evidently far removed from the cs beliefs of students today, except for the notion that gravity is a tendency for heavy objects to fall down. 
However, many students share with Aristotle the belief that the speed of a falling body is proportional to its weight $W$. Like Aristotle, their only measure for motion is average speed. So the speed $V$ of an object which has fallen a distance $D$ from rest in time $T$ is given by

$$
V=D / T
$$

Aristotle discussed the resistance of a medium in detail and inferred that the speed is inversely proportional to the resistance $R$, which depends on the size and shape of the body as well as the density of the medium. Thus in an appropriate system of units (which Aristotle never discusses), Aristotle's law of falling bodies can be written as

$$
V=W / R
$$

Consequently, for two bodies of the same size and shape released simultaneously from rest, at any time the ratio of speeds is given by

$$
v_{1} / v_{2}=D_{1} / D_{2}=W_{1} / W_{2}
$$

In other words, the heavier body falls faster (farther) in proportion to its weight. Of course, Aristotle's law is not true, but neither is Galileo's law (for bodies falling through a medium).

As a historical caveat, it should be mentioned that Aristotle's physics was qualitative rather than quantitative. Indeed, he believed that quantitative physics is impossible. Nevertheless, Eqs. (1) and (2) are straightforward algebraic formulations of his qualitative assertions, and the relations expressed by Eq.(3) were inferred and discussed by his followers in the middle ages.

Let us turn now to the other kind of force in Aristotle's theory. An external force can be exerted on an object only by a living agent in direct contact with it or indirectly through some connection such as a rope. Nonliving things are obstacles that stop or guide motion, but they do not exert forces. A force does not move an object unless it overcomes (exceeds) the object's inertia, an intrinsic resistance (mass) which is not distinguished from weight. A constant force $F$ imparts to the object a constant speed $V$, which is inversely proportional to the resistance $R$, depending on the medium as well as the object's inertia. In algebraic form unknown in Aristotle's day, this can be written as

$$
V=F / R \text {. }
$$

In accordance with this law, followers of Aristotle concluded that an increase in speed (acceleration) can be achieved by an increase in the force, or, in the case of natural motion (free fall), by an increase in weight as the object gets closer to its natural place.

Aristotle supposed that in the absence of any force an object comes to rest immediately. So, to explain such phenomena as the flight of an arrow, he was obliged to attribute motive as well as resistive powers to a medium. Thus the arrow is propelled by the air collapsing in behind it and so transmitting, of course, the force exerted by the living agent who drew the bow. Criticism of this ad hoc argument lead to a revision of Aristotelian theory in the middle ages, to which we now turn.

\section{IMPETUS PHYSICS}

Aristotle's idea that a medium has motive power was introduced to explain the persistence of motion in the absence of an external agent, in accordance with the general principle that every motion has a cause. This idea was rejected in 
favor of an alternative explanation by Johannes Philoponus of Alexandria, a philosopher and commentator on Aristotle. He proposed that when an object is thrown, the active agent imparts to the object a certain immaterial motive power which sustains the body's motion until it has been dissipated due to resistance by the medium. This transmitted motive power was called impetus by Jean Buridan, who was responsible for the definitive formulation of impetus theory in the 14 th century.

Impetus is such a natural CS concept that some version of it occurs to a substantial fraction of students today, ${ }^{3}$ who usually refer to it as a force and tend to confuse it with the Newtonian force concept. Buridan's formulation of the impetus concept is such a clear articulation of the more or less vague intuitions common among students that it is worth quoting ${ }^{15}$ :

\begin{abstract}
"A mover, while moving a body, impresses on it a certain impetus, a certain power capable of moving this body in the direction in which the mover set it going, whether upwards, downwards, sideways or in a circle. By the same amount that the mover moves the same body swiftly, by that amount is the impetus that is impressed on it powerful. It is by this impetus that the stone is moved after the thrower ceases to move it; but because of the resistance of the air and the gravity of the stone, which inclines it to move in a direction opposite to that towards which the impetus tends to move it, this impetus is continually weakened. Therefore the movement of the stone will become continually slower, and at length, the impetus is so diminished or destroyed that the gravity of the stone prevails over it and moves the stone down towards its natural place."
\end{abstract}

Note that this statement includes the possibility of circular impetus, which Buridan used to explain the persistent motion of the planets and the grinder's wheel. Note also that the impetus idea can account for motion in a vacuum, and so overcomes Aristotle's main arguments against the possible existence of a vacuum.

The impetus concept is a historical precursor of the concepts of momentum and kinetic energy. It had a significant influence on Galileo's thinking, as emphasized by clement ${ }^{2}$ in a pedagogical context.

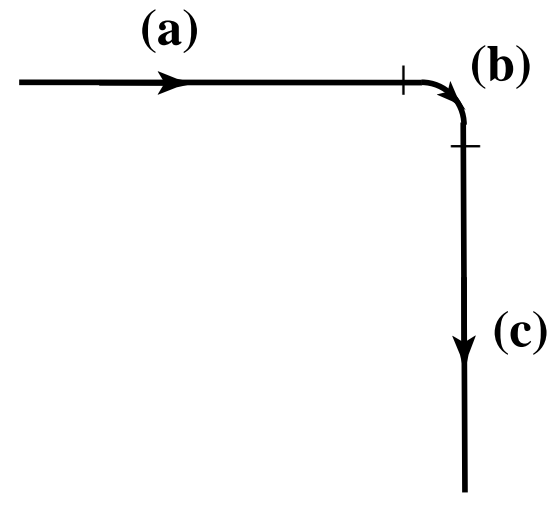

Fig. 1. Albert of Saxony‘s projectile path.

Albert of Saxony used Buridan's theory to explain projectile motion. This involves a compromise between the effects of impetus, gravity, and air resistance. For a projectile launched horizontally, Fig. 1 shows the threestage trajectory he drew. ${ }^{16}$ As he explained, in the initial stage (a), the impetus suppresses any effect of gravity and propels the projectile horizontally until it is sufficiently weakened by air resistance. The intermediate stage (b) shows a compromise between impetus and gravity until the initial impetus is exhausted, and in the final stage (c), the projectile falls 
vertically in "natural motion." A similar reasoning is exhibited by students in this age, as we shall see.

Several important kinematical ideas were developed in the 14th century along with impetus theory: (a) a clear distinction was made between uniform velocity, uniform acceleration, and nonuniform acceleration, which gave birth to the concepts of instantaneous velocity and acceleration. This shows that a qualitative understanding of these concepts is possible without mathematical formalism, and thus suggests a worthy pedagogical objective. (b) Oresme invented the graphical method for representing variable quantities. (c) This was used to derive the Mertonian "mean speed rule," which says that in a given time interval, the distance traveled with uniform acceleration is equal to the distance traveled with a uniform speed equal to the instantaneous speed in the accelerated motion at the middle instant of the time interval. All these ideas were essential prerequisites to Galileo's kinematical analysis of projectile motion. We have better symbolic means for representing these concepts today, but history helps reveal the difficulties in establishing a firm intuitive base for the concepts.

\section{COMMON SENSE CONCEPTS OF COLLEGE STUDENTS}

To survey concepts about motion held by college students enrolled in physics courses, we used a multiple-choice mechanics diagnostic test and conducted interviews with a sample of 22 students within one month after they had taken the test. A copy of the entire diagnostic test is displayed in the Appendix to the preceding paper, ${ }^{1}$ and we will frequently refer to specific tasks on the test in the discussion below. The conditions under which the test was given and average performances of students were reported previously. Here we analyze the responses to specific questions. All statistics reported below are for a group of 478 students in university physics. Pretest (post-test) results were obtained by administering the diagnostic test at the beginning (end) of the semester.

The multiple-choice alternatives to a number of the questions on the diagnostic test are readily classified as characteristic of either Aristotelian, Impetus, or Newtonian theories. This enabled us to classify pretest responses as predominantly Aristotelian for 18\% of the students, predominantly of the Impetus type for 65\% of the students, and predominantly Newtonian for the remaining 17\%. However, nearly every student used some mixture of concepts from the three theories, and appeared to be inconsistent in applying the same concept in different situations. For example,

(a) On the pretest (post-test), 47\% (20\%) of the students showed, at least once, a belief that under no net force, an object slows down. However, only $1 \%$ (0\%) maintained that belief across similar tasks.

(b) About 66\% (54\%) of the students held, at least once, the belief that under a constant force an object moves at constant speed. However, only 2\% (1\%) held that belief consistently.

(c) About 65\% (44\%) of the students exhibited, at least once, the belief that an impetus is required to maintain the motion of an object. About 40\% (24\%) were consistent in that belief. About 37\% (15\%) maintained, at least once, that the trajectory of an object depends on an impressed impetus, but only 3\% (1\%) were consistent in this belief. Students with quasi-Newtonian beliefs were far more consistent than the other students.

The CS conceptual systems of the students have much less internal coherence than the Aristotelian and Impetus systems. They can best be described as bundles of loosely related and sometimes inconsistent concepts. For example, although $84 \%$ of the pretested students believed that a free particle follows a linear trajectory, only 30\% believed that the speed of such a particle is constant. Moreover, only 15\% of the students held the Newtonian belief that under a constant force a particle has constant acceleration.

No doubt much of the incoherence in the student CS systems is the result of vague and undifferentiated concepts. On the pretest, $82 \%$ of the students 
believed that intrinsic geometrical and physical properties of an object affect its free fall in a vacuum. Nearly all these students held mixtures of Aristotelian and Impetus beliefs. Of these students, 83\% failed to recognize the height symmetry in task (X) of the diagnostic test, and 61\% confused the concepts of position, speed, and acceleration at least once. Such misconceptions were rare among students who knew that the intrinsic properties of an object do not affect its free fall.

To probe common sense beliefs more deeply, 22 students were interviewed at length about their responses on the diagnostic test. The students invariably reiterated the answers they had given previously and were slow to change them as the questions were discussed in greater depth. The students were asked to justify their answers and opinions, and the interviewer repeatedly introduced contrary information and asked for comparisons between different physical situations in an effort to test the stability of the students' beliefs.

During the interviews with several of the students, typical classroom demonstrations were given of the physical situations described in a few of the talks on the diagnostic test. The demonstrations appeared to have no more effect on their opinions than mere discussions of the phenomena. As a rule, students held firm to mistaken beliefs even when confronted with phenomena that contradicted those beliefs. When a contradiction was recognized or pointed out,

they tended at first not to question their own beliefs, but to argue that the observed instance was governed by some other law or principle and the principle they were using applied to a slightly different case.

One student argued that in task (IV), the ball after release starts its motion in a circular arc before it takes on the correct parabolic path. After watching the motion of a ball in air released from an electromagnetic arm rotating uniformly in a vertical circle, he maintained that, despite seeing the ball follow a parabolic path, the "ball will swing the same way as before it was disconnected for a short while... [that] we cannot see with the naked eye."

Another student had argued for a straight path on question (26) of task (IX). After watching a simulated parabolic motion of a puck on an air table, she argued that "if the table were long enough, the puck would eventually go straight across ... maybe even in the direction of the air [blown from a hose on the puck]... after the force due to the air overcomes the initial velocity that would take the puck in this direction [that of the rocket before the engines were fired]."

Careful interviews of students who have just witnessed a demonstration are enough to make one dubious about the effectiveness of typical classroom physics demonstrations in altering mistaken physical beliefs. We doubt that a demonstration can be effective unless it is performed in a context that elicits and helps to resolve conflicts between common sense and specific scientific concepts.

After long discussions, most students who showed obstinate beliefs were able to come to adequate justifications, mostly not because they have seen something in conflict with their beliefs, but because they came to realize the inconsistency of their thinking when asked to reflect on their own arguments.

To survey the variety of student concepts in an orderly fashion, we have catalogued our results in Sec. IV A-D which follows.

\section{A. General concepts about motion}

This subsection is concerned mainly with how students "define" their concepts of force, gravity, and motion. Subsequent subsections will be concerned with how they use the concepts to explain dynamical effects.

Most of the interviewed students had acquired a rote knowledge of Newton's laws, either from the physics course in which they were enrolled at the time, or from a previous course. They could enunciate Newton's laws on request, but usually they were unable to see how the laws applied to a particular question. When asked to justify why a projectile follows a parabolic path, one student replied, "I don't know. I have been taught that it goes in a parabola, but I never understood why." 
In everyday life, the term "force" is used in a chaotic variety of context-police force, economic force, force of argument-often with vague and ambiguous associations. Thus it is to be expected that beginning students are prone to use the term "force" loosely for a variety of different concepts, some of which are not even dynamical. One student asserted that "acceleration is a force. It sounds like a force." On the pretest, $65 \%$ of the students maintained the prescientific belief that "every motion has a cause." In their search for a cause of motion, interviewed students gave the following names to a purported cause:

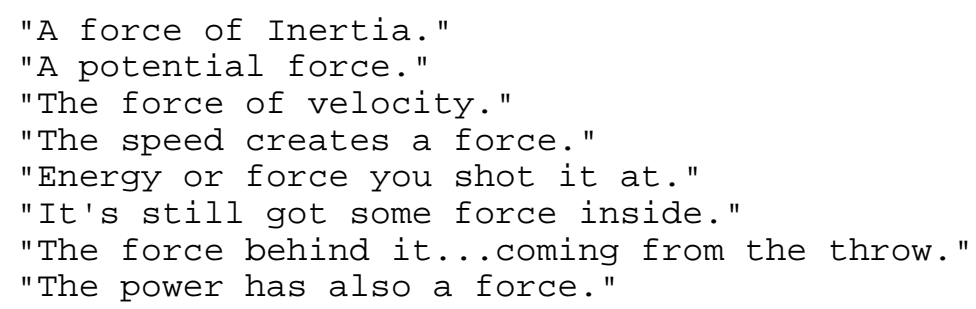

Some students compared the magnitude of a force to the magnitudes of kinematical concepts:

"The speed is equal to the force of pull."

"The initial velocity is greater than the force."

"The energy of blast has to be greater than the force."

Students did not use a Newtonian classification of forces. Rather, they distinguished a force that:

"only starts the motion," or

"is just changing the direction of motion," or

"has nothing to do with [changing] the speed, it only has to keep the ball moving."

For some students, the effect of a force may not appear at the instant it is applied, or the effect may be self-consuming or dissipated by external resistances:

"The force does act only after...it overcomes the initial velocity."

"This force cannot stay forever... Nothing stays forever."

"The cannon has enough force to take [a cannonball] only that far."

"The force decreases...because of the pull of gravity in that [opposite] direction."

Many students believe that inanimate objects may serve as barriers to stop or redirect motion, but not as agents of a force. As one student explained,

"There was a force when you were holding [a ball] in your hand... [but when the ball is sitting on a table], there is not a force on the ball...this is different. The ball wants to go down, but the table is only holding it...keeping it from moving."

The same student argued that a force is not required for objects to fall, since they "always want to go down." As he explained,

"there is no force on the ball [falling down]...There was a force when you were holding it, but when you let it go, there is no more force and the ball is free to fall...the ball wants to go down, but you were holding it...so when you let go, it goes back to ground, and there is gravity." 
Here the interviewer intervened to ask whether gravity is a force; the student replied:

"No. I don't know...I guess not since [after a ball is released] it speeds up for a short while...to reach the speed of fall. Then the ball must go at constant speed because there is no force to stop it or maybe to make it speed up....So I guess no, gravity is not a force."

Some students believe that gravity is a kind of impetus acquired by falling objects. As one student said, "gravity increases as objects fall...because their speed gets greater and greater." We shall hear more of this idea later.

All the interviewed students accepted the existence of a vacuum, but some maintained that motion is impossible in the absence of a material medium. As one student explained,

"If you release a body in a vacuum, it stays where it is...There is no motion in a vacuum...because gravity does not act in a vacuum. Gravity acts only if there is air...a body moves down because of the air that pushes down on the body...Air pushes also in all directions; this is friction...But the push down is greater than friction and that is why a body falls."

"The heavier a body is, the less the effect of friction...The mass of a body helps air push down. . . [In water, the speed of fall is smaller than it is in air] because friction in water is greater than in air. The water pushes down more than air, but the speed is smaller because friction in water has a larger effect than in air [because of the density of water], larger than the push down."

With respect to the kinematical aspects of motion, the most common and critical problem for students was a failure to discriminate between the various kinematical quantities. This was clear among the interviewed students, although most of them had already completed a study of kinematics in their physics classes. Over 30\% of the students maintained on the pretest that two particles have the same speed when they simultaneously occupy the same position, even if the two particles were moving with different constant speeds. When interviewees failed to justify or refute their misconception, they were asked to assume that the two particles [task (I) of the diagnostic test] move with constant speeds of 10 and $15 \mathrm{mph}$, respectively. Then they were asked what the common speed of the two particles would be at the instant they met. A student replied:

"Since they met at [position 2 of task (I)], both should have the same speed... It is a value between 10 and $15 \mathrm{mph}$." [When reminded that the two balls move with constant speeds], "then I guess they cannot have the same speed. [Ball] A will always go at $10 \mathrm{mph}$, and B at $15 \mathrm{mph}$. But when they are at [position] 2, how could they meet if they don't have the same speed? Wouldn't they, at least at the instant they met?"

Faced with the same problem, two other students argued,

AL: "Since they met...they meet during a certain period, no matter how small it is...during that period, they are at the same position, they must have the same speed, otherwise how could they have met!"

SC: "Their speed would be $13 \mathrm{mph}$ or so...but they have constant speeds...B covers a longer distance than A, but they both reach there [position 2] at the same point... and during the same time...oh, no! They should have different speeds at $2 . "$

The rest of the protocol for $\mathrm{SC}$, which we do not relate, provided a beautiful example of intellectual equilibration, as she recognized a contradiction in her 
thinking and went on, with some help by the interviewer, to discriminate between average and instantaneous speeds.

\section{B. Free particle motion}

Students with Aristotelian beliefs produced the most bizarre arguments. Thus one student argued that block X of task (XI) would instantaneously come to rest after external forces cease to drive it because "you need wheels to keep it going." Arguments furnished by Impetus thinkers were more plausible. Students GT and ST had shown straight paths for the ball leaving the tube of task (V). However, they had two typical erroneous beliefs about the kind of speed the ball would have along the path:

GT: "...the ball before wanted to go in a straight line, but because [the tube] doesn't let it go, the ball acquires a power that will make the ball jerk [when it leaves the tube] and acquire a new speed bigger than the one it had before... This power depends on how long the ball twirls inside the tube...It will let the ball speed up until it wears out. Then the ball goes at constant speed."

ST: [When the ball moves inside the tube], "it acquires a power waiting to be released. The speed of rotation [inside the tube] creates a force or a power ...that makes the ball first speed up, then after the power vanishes, [the ball] starts decelerating."

Most Impetus students believed that the impetus starts wearing out at the instant the ball leaves the tube, while some maintained that this impetus is maintained until some resistance is encountered. For that impetus, students used the names power, force, acceleration, velocity, momentum, inertia, or energy indiscriminately.

Two other students, AC and SC, had shown curved paths for the ball of task (V), and argued that the motion is maintained by a sort of circular impetus.

AC: "When you train something to do something for quite some time...this thing [the ball] will do the same thing [move in a curved path] it was trained to do, by itself."

SC: "The ball goes like this [curved path] because it still had some momentum when you were turning it in a circle, and it wants to go in a straight line. So it does not go in a circle [back inside the tube] and not in a straight line. Instead, it goes [in a curve] until the momentum wears out...then it goes straight."

A compromise between the "natural tendency" of the ball to go straight and the motion it was trained to undergo was shared in a different way by less than 1\% of the students. Those students argued that as a result of the circular motion inside the tube, the ball of task (V) acquires a tendency to move radially outward, i.e., to leave the tube in a centrifugal direction. The actual path the ball follows outside the tube will be a compromise between the acquired centrifugal tendency and its natural tangential tendency. A student who had shown such a compromise path on the pretest explained,

"The ball goes out in the direction of the resultant of the acceleration of rotation [the tendency to move tangentially to the tube] and the centrifugal force that would take the ball [radially outwards]."

Incidentally, the same student had argued that if the ball of task (V) was to leave a tube suspended in air, but in the absence of gravity, the ball would "stay in place. It does not move beyond the point you left it at." Yet, when the tube is laid on a horizontal table, the linear motion of the ball outside 
the tube becomes possible due to the presence of a rigid support, or guide: the table. On the other hand, the student argued that linear motion in thin air could have also been possible outside the tube, or beyond the edge of the table, if the tube or the table on which the tube lies were "long enough to...train the ball to go that way."

A kind of impetus conservation was shown by 28\% of the students on task (IX) of the pretest. Those students believed that in the final stage of the rocket's motion-after the engines are shut off-the rocket would return to the direction of motion it had before the engines were turned on. Interviewed students who showed such a belief argued that when the rocket was coasting in space in the absence of external forces, it was endowed with a kind of impetus that took it in the specified direction. When the engines are turned on, they continued, a force acts against that impetus and takes the rocket in a compromise direction. "When you shut the engines off," a student argued, "there is only the horizontal component [the one parallel to the initial direction] of the speed, and the rocket goes in its direction." Thirteen percent of the students had argued that in the second stage (when the engines are on) the rocket acquires a new impetus that adds up to the former one in the final stage. As a result, the rocket moves in the direction of the resultant of the two impetuses after the engines are turned off.

Students with Impetus beliefs differed not only with respect to how the speed and direction of impressed motion are maintained, but also with respect to how an impetus is acquired, and to whether and how it dissipates. These differences are better revealed in Sec. IV C and D.

\section{One-dimensional motion under a constant force}

In this subsection we examine student beliefs about motion under a constant force, including gravitational free fall and fall constrained to inclines. On the pretest, $14 \%$ of the students shared the belief that a particle subjected to a constant force moves with a constant speed. Of the students who maintained that under a constant force the speed of a particle continuously increases, 40\% believed that the increase in speed is proportional to the magnitude of the force and the distance travelled. Of all students, 47\% believed that the time interval required to travel a specified distance under a constant force is inversely proportional to the magnitude of the force. None of the students showed the Aristotelian belief that a constant motive force moves a physical object only a limited distance. But 27\% of the students held that a force cannot keep accelerating an object indefinitely, and that the object reaches a critical speed limit determined by the magnitude of the force and the mass of the object.

With respect to task (XI), student $\mathrm{CM}$ argued that "if the mass of block X is greater than the force of [pull] of $Y$, block X stays in place...it could not be moved." For CM, as for Aristotle, the mass of an object is a resistive force even on frictionless surfaces. Students who shared this belief were asked during the interviews to imagine block $x$ pulled on different surfaces, e.g., polished and unpolished wood surfaces, sand, ice, etc., and to compare the motions that the block would undergo on the various surfaces. CM, like a few other students, maintained that the mass of the block always resists motion the same way, except on surfaces like ice, but only because "ice is slippery."

Students who believed that a particle under a constant force always reaches a speed limit furnished diverse arguments for their belief.

GT: "Block $X$ first speeds up until it reaches a speed equal to the pull of Y...then it keeps going at that speed... That maximum speed is always equal to the force you apply. [For instance, if you apply a force of 10 lbs. to block $\mathrm{X}]$, the maximum speed would equal $10 \mathrm{ft} / \mathrm{s} . "$ 
BM: "A constant force speeds up a body...but only until the body reaches the speed that would have used all the power of the force. [The body thereafter moves at constant speed]. "

AP: "Isn't there a limit for everything?...How could an object go faster and faster all the time...there must be a limit."

PA: "Gravity pulls down [on an object in free fall], but there is something that makes it level off. I don't know what it is. It just does not seem reasonable that its speed can keep increasing indefinitely."

AL: "Galileo did the [free fall] experiment in Pisa and said they [falling objects] reach a speed limit. I guess...because Galileo did it, or at least if what I know about him is true, this must be true."

KC: "I answered there is a maximum speed [in free fall] because I based my answer on what a friend told me. He is a parachutist. He told me that because of air, they can reach a speed limit."

The last two statements remind us that students are all too ready to justify their beliefs by an appeal to authority, one of the achievements of teaching passive rote knowledge.

Seven interviewees believed that after block $X$ of task (XI) reaches its maximum speed, that speed will be maintained whether or not block Y remains connected to block X. Furthermore, some believed that changing the magnitude of the motive force does not change the magnitude of the speed limit. For instance, GT argued that if you "double the pull on block X [the maximum speed] remains the same, only the time... and the distance...to reach that maximum will be cut in half."

Six interviewed students who had argued for a maximum speed in free fall said that this speed "is equal to gravity...32 ft/s." On the other hand, some students believed that "gravity does not act instantaneously from the moment [you set an object free to fall], it takes a while." Js and CM gave similar arguments:

JS: "Zero force acts on the ball [at the instant it is released]. As it goes down, the force of gravity increases... and that's why the speed increases."

CM: [A free falling ball] "goes faster and faster because gravity pulls more and more as it goes down. It's just like a magnet. The closer the object is to the magnet, the harder it is attracted... Gravity pulls harder, the closer the ball comes to earth."

On the other hand, some students believed that gravity does not act the same way in free and constrained fall. The distinction, though, is not clear. Some students, like PD, believed that in free fall, but not on inclines, objects move with the same acceleration irrespective of their physical properties.

PD: "Heavier bodies slide down an incline faster than lighter ones...even if friction and air resistance are ignored... On inclines, gravity does not act the same [as in free fall]...there is a difference somehow, but I don't know what it is."

For some students, gravity manifests itself by a constant force but not by a constant acceleration. Objects of different mass then cannot fall with the same acceleration.

CM: "Gravity means the same force on any object...it pulls as hard...the same way...I mean by an equal force on all objects." 
PM: "Gravity means the same force pulls on different objects...I know that gravity is 9.8 for all bodies."

JM: "Acceleration of gravity is constant. Then if you double the mass...and since you have the same acceleration...two units of mass go half as fast as one unit of mass."

Some students even believed that weight and "gravity" are two different forces. $\mathrm{KB}$ argued that "The velocity [of free fall] is due to the weight and gravity. It is due to the resultant of both." For students like kB the weight increases with the mass of a falling object, whereas "gravity is constant for all" objects. Or, another student says,

PA: "Heavier objects fall faster because I know this...Because we have two kids... and when we go down a water slide which is reasonably frictionless...I go faster when I have a kid on my shoulders than when I go down alone...Gravity is the same for all objects... It's the same pull all the way around for different objects... But beside gravity there is the weight....

[Yet if two objects of different masses fall together], the heavier one falls faster first until a certain point...then the other one catches up and they level off... and they go together at the same speed all the rest of the time."

This "catching up" process is better revealed in Sec. IV D. As to why it happens, students could not provide any better explanation than "I know it just happens", or, "it just seems logical."

\section{Two-dimensional motion under a constant force}

Many students have some notion of parabolic motion, but few of them recognized it as the consequence of a constant force. Thus 66\% of the pretested students were able to identify the correct parabolic path for the projectile of task (VI), but only 20\% of those students were able to identify the similar path for the rocket firing its engines in task (IX). Interviews confirmed that the students had great difficulty identifying a common principle in the two tasks.

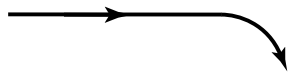

(a)

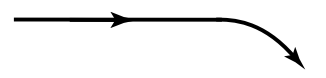

(b)

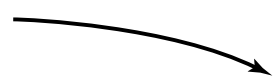

(c)

Fig. 2. Projectile paths depending on how an impetus was imparted: (a) for an object thrown by hand, (b) for an object released from an airplane, and (c) for an object projected off a table.

Most of the students maintain impetus concepts, but differences in their concepts are evident in their comments about projectile motion. Some of them believe that a projectile's motion is not only determined by its initial velocity, but also by how that velocity was imparted. JT asserted that a ball launched in the air with an initial velocity $v$ goes horizontally for a while, traveling a distance that is greatest if the ball is thrown by hand, smaller if released from an airplane flying with that velocity, and much smaller if projected off a table with velocity $V$ (Fig. 2). Another student who argued for the same paths tried to explain the difference.

AC: "[In case (a)] you are giving the ball a speed in a straight direction...the harder you throw it, the more it will go straight. [In cases (b) and (c)] you are not giving it power, you did not give the ball power to go 
in a straight line first as you did [in case (a)]. Here, [case (a)] you're giving it an energy, a certain energy to go straight...there, because the plane was just carrying it [and because the table was directing the ball]...the plane does not give it [the ball] a power to go straight."

Students RS and DL argued that the ball of Fig. 2 goes as shown in cases (a) and (c), but not in case (b). In the latter case, the ball would go straight at an angle with the horizontal, as shown in Fig. 3.

DL: [The path followed by the ball] "is the resultant of the velocity given to it by the plane and gravity... [but if the airplane's engines suddenly are turned off, or the ball is thrown by hand or projected off a table] the plane/ball goes like this [Fig. 2(a)] because the horizontal velocity overcomes gravity...the horizontal force which is a product of the blast...is greater than gravity... here [when the ball is released from the flying plane] this ball is just dropped ...first it was carried by the plane and it is just dropped. It's not given any blast or anything."

AL: [Argued that a projectile falls straight down unless launched with a velocity whose magnitude is above a certain critical level, no matter at what angle you launch that projectile. Furthermore, he argued that no matter how you launch a projectile, it never starts its motion tangentially to the initial velocity, and that is] "because gravity is pulling down on it."

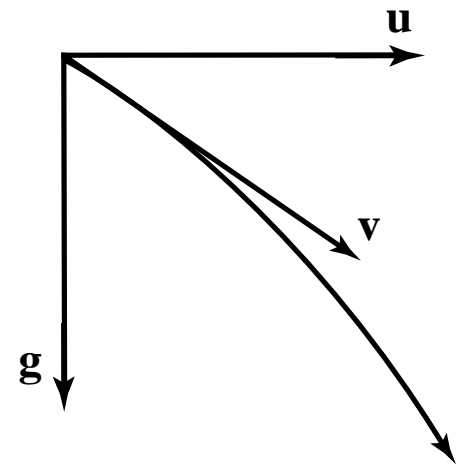

Fig. 3. "Compromise" velocity $\mathbf{v}$ and path of a projectile released with initial velocity u subject to "gravity $\mathbf{g} . "$

Some students, like RS, argued that the projectile could start the motion in the direction of the initial velocity, but only if that velocity is "greater than gravity" (Fig. 4).

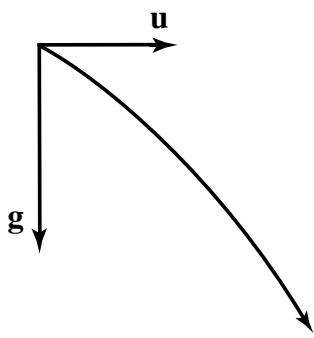

(a)

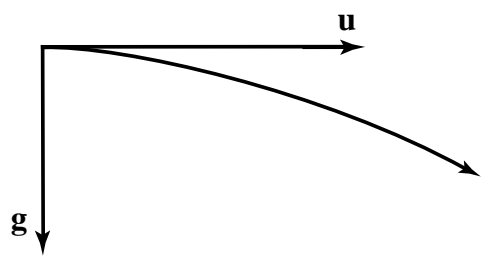

(b)

Fig. 4. Path of a projectile subject to "gravity g" and released with initial velocity u. (a) "smaller than or equal to $\mathbf{g}$," or (b) "greater than $\mathbf{g . "}$ 
Students who argued for the paths of Fig. 2 were divided as to how the impetus varies. Some, like RS, maintained that the impetus, hence the speed of the impressed projectile, remains constant along the horizontal part of the path. Others, like JS, maintained that the impetus starts wearing out from the instant an impressed projectile gets disconnected from the original mover. Consequently, the speed of the projectile decreases along the straight part of the path that is in the direction of the initial velocity.

RS: "Its speed remains constant...because of the power...because of the force behind it...until the power starts wearing out...and the initial velocity is overcome by gravity. Then [the ball starts curving and] its velocity keeps increasing because of $g$...It keeps curving until the power vanishes [or as AL put it, "until the horizontal velocity becomes zero"]...then gravity takes over... and the ball falls straight down at constant speed."

JS: "The ball goes first in a straight line because of the force behind it...This force is constant... Oh no! It can't be, because it slows down here [along the horizontal part of the path]. Force, then, must decrease up to here [where curving starts], because gravity acts down on it...from here on [straight vertical part of the path] there is no more force behind it...only gravity pulls it down."

Some students, like SL, maintained that the impetus remains constant, or like TS, that the impetus can build up during the motion.

SL: "Because of the amount of energy or force you shoot it at...the thrust of the firing keeps the ball going in a straight line...But gravity pulls down on the ball...it pulls more and more...until [gravity] equals this amount of force [impetus]. The ball then starts curving down...it keeps curving because of the amount of energy that is still propelling it forward...but gravity is now becoming greater and greater."

TS: "The force [impetus] increases as the ball goes down, because gravity is pushing down now in the same direction of the motion...then it provides the ball with more and more force as it goes down...but gravity, the pull of gravity is constant."

Arguments similar to the above were given for projectiles launched at an angle with the horizontal (Fig. 5).

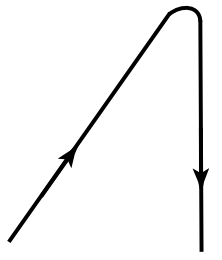

(a)

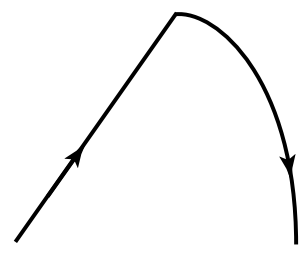

(b)

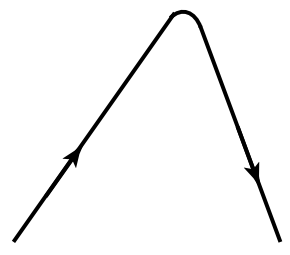

(c)

Fig. 5. Typical impetus paths for projectiles launched in an oblique direction

JT: [On the way up], "the ball goes straight up because of the force...that the ball receives when you fire it...This force decreases on the way up...but the ball moves at constant speed... The force has nothing to do with the speed, it only has to keep the ball moving... [On the way down] you don't need that force any more...The ball would fall down, I mean always fall down if there is no 
force. So there must be a force that moves the ball up. But this force cannot remain the same because the ball wants to go down....and this takes a power from the force, so it diminishes [to a value that is not big] enough to take the ball up...but enough to take it sideways instead of [falling straight down]."

For JT, that ball maintains a constant speed during all its motion.

RS: [Around path (c) of Fig. 5], "the force behind it diminishes...but not completely... The ball falls at constant speed...the initial power tends to take the ball [horizontally] but gravity pulls it down [vertically] so that the path [on the way down] is the resultant of both [impetus and gravity].

For most students, an impetus maintains motion in the direction of the velocity of projection or in the direction of the resultant of that velocity and other existing forces. However, for 15\% of the students an impetus also maintains the path of motion, as if an impressed object "trained to do something" gets endowed with a memory, or becomes conditioned "to do what it is trained to do."

\section{A TAXONOMY OF COMMON SENSE CONCEPTS ABOUT MOTION}

To organize our knowledge about CS concepts and provide a guide for applying it to instruction, in this section we develop a brief annotated taxonomy of the most significant CS concepts. It would be impractical to categorize the many variations of each concept found among students, so we have attempted to give formulations of the concepts which express the most common beliefs.

To develop a taxonomy, we need classification principles. Fortunately, Newtonian mechanics provides us with a ready-made classification scheme, and we can classify CS concepts about motion as alternatives to specific Newtonian concepts. Accordingly, we recognize two general categories: principles of motion, corresponding to Newton's Laws of Motion, and influences on motion, corresponding to specific laws of force in Newtonian mechanics.

\section{A. Principles of motion}

(1) Description of motion: CS kinematical concepts commonly have the following characteristics.

(a) The concepts of "time interval" and "instant of time" are not differentiated. An "instant" is regarded as a very short time interval.

(b) Velocity is defined as distance divided by time. Thus average velocity is not differentiated from instantaneous velocity.

(c) Concepts of distance, velocity, and acceleration are not well differentiated.

(2) In the absence of forces, every object remains at rest (with respect to the earth). In a common sense system, this principle plays a role analogous to Newton's First Law.

The tacit adoption of the earth as a preferred reference frame is especially significant, as it is undoubtedly based on direct perceptual experience. One of the marvels of the human perceptual system is the fact that from diverse sensory input it creates a representation of an environment at rest while the observing subject moves, rather than one in which the observer is always at rest while the environment moves. Of course, the testimony of the senses is not to be denied, rather, Newtonian theory tells us how it should be reinterpreted to be consistent with a wider range of experience. This example suggests that to deal most effectively with particular cs beliefs, instructional design should depend on how those beliefs are grounded in perception, but that is a matter for future research. 
(3) The causal principle of motion: Every motion has a cause. This is a CS analog of Newton's second law.

(a) Motion is started by

(i) a force applied to the object by an external agent;

(ii) Gravity, an intrinsic tendency to fall down.

(b) Motion is sustained by

(i) continuous action of an applied force or gravity,

(ii) an internal force (Impetus) in the object.

(c) Motion may be opposed by

(i) intrinsic resistance (weight or mass) of the object,

(ii) resistance of a medium surrounding the object,

(iii) obstacles that "get in the way."

The action of a resistive medium or an obstacle is not an active force, because it does not start or sustain motion. It may, however, be called a reactive force, to help students develop a general force concept.

(4) Newton's third law is inconsistent with common sense intuitions. Maloney ${ }^{17}$ has studied and classified rules generated by students to deal with situations where the third law applied. He found that most students characterize the reciprocal interaction between two objects by some sort of dominance principle: (a) The greater mass exerts the greater force, or more frequently, (b) the object which causes motion of the other exerts the greater force, because it overcomes the other's opposition.

(5) The Newtonian superposition principle (Vector addition of forces) has two CS analogs:

(a) Dominance: Motion is determined by the larger of two competing forces. This principle has a natural origin in the experience that, to move a heavy object, one needs to push harder and harder until the push "overcomes" the resistance, and less effort is needed to maintain motion. A student needs to reinterpret this experience to accept Newtonian theory. Textbook statements such as "resistance can be neglected," might be interpreted by students as confirmation of the dominance principle.

(b) Compromise: Motion is determined by a compromise among competing forces. Of course, the superposition principle can be regarded as a kind of compromise, but student ideas of compromise are likely to be vague or involve an impetus. Sometimes dominance and compromise principles are used together, as we saw in connection with Fig. 4.

\section{B. Influences on motion}

(1) An applied force is a push or pull exerted by an agent in direct contact with the object. For some, only living things are recognized as agents of force. The effect of an applied force is commonly characterized by the following causal principles:

(a) Inertial resistance: A force cannot move an object unless it is greater than the object's weight. Weight is not distinguished from mass.

(b) A constant force produces a constant velocity, sometimes expressed as $F=m v$.

(c) Acceleration is due to increasing force.

(d) A constant force has a limited effect depending on its magnitude. The limitation may be one of two kinds:

(i) The force wears out, due to its consumption by the motion or its dissipation by resistive agents. Furthermore, its effect may not be instantaneous, in the sense that the effect may not start until sometime after the force is applied.

(ii) The force $F$ accelerates the object until it reaches a critical speed proportional to $F$, which the object maintains afterwards whether or not the force is still applied. 
(e) A long-range force must be transmitted by a medium, such as a rope connecting object and agent. Therefore, long-range forces cannot act on an object in a vacuum.

(2) An internal force (or impetus) maintains motion of an object independent of external agents. As Clement ${ }^{2}$ has observed, by this principle students frequently infer the existence of a force in the direction of an object's motion.

(a) An impetus can be imparted by an applied force and transmitted from one object to another.

(b) The impetus of an object is proportional to its mass and velocity, as expressed by the equation $F=m v$.

(c) An impetus may wear out or build up in the same way as the effect of an applied force.

(3) Resistance opposes an applied force or consumes the impetus of a moving object. The following kinds of resistance are not always distinguished:

(a) Inertia (weight or mass) is an intrinsic resistance of an object to motion.

(b) Friction due to contact with a solid surface.

(b) Fluid resistance depends on the density of the fluid as well as the size, shape, and weight of the object.

(4) Obstacles may redirect or stop motion, but they cannot be agents of an applied force. Minstrel ${ }^{15}$ has analyzed student concepts of reactive forces.

(5) Gravity is a tendency of objects to fall down. In this conception, gravity is not necessarily a force. Nevertheless, the causal principles for applied forces which we noted above may be attributed to gravity as well. As we saw in our discussion of Aristotelian physics, an important consequence of those principles is the belief that heavier objects fall faster. This belief is so common that it deserves to be examined carefully in physics classes.

In preceding sections we noted a number of other beliefs about gravity, and more are noted by Gunstone and White. ${ }^{6}$ But more important than particular beliefs about gravity may be the uncertainty of students about what gravity "really is." So the best teaching strategy may be the direct one that aims at convincing students that gravity is a force, in particular a long-range force. The idea of a long-range force is difficult for students to understand and accept, as it was for many great intellects in history. Historically, Gilbert's study of magnets did the most to convince people of the reality of long-range forces. Physics instructors may draw a pedagogical lesson from this.

a) Now at the Lebanese University II.

1. I. A. Halloun and D. Hestenes, "The initial knowledge state of college physics students," Am. J. Phys. 53, 1043 (1985).

2. J. Clement, "Students" preconceptions in introductory mechanics," Am. J. Phys. 50, 66 (1982).

3. M. McCloskey, "Intuitive physics," Sci. Am. 249 (April), 122 (1983).

4. M. McCloskey, A. Caramazza, and B. Green, "Curvilinear motion in the absence of external forces," Science 210, 1139 (1980).

5. J. Minstrell, "Explaining the 'at rest' condition of an object," Phys. Teach. 20, 10 (1982).

6. R. F. Gunstone and R. White, "Understanding gravity," Sci. Ed. 65, 291 1981).

7. L. Viennot, Eur. J. Sci. Ed. 1, 205 (1979).

8. D. E. Trowbridge and L. C. McDermott, "Investigation of students' understanding of the concept of velocity in one dimension," Am. J. Phys. 48,1020 (1980); "Investigation of students' understanding of the concept of acceleration in one dimension," Am. J. Phys. 49, 242 (1981).

9. A. B. Champagne, L. E. Klopfer, and J. H. Anderson, "Factors influencing the learning of classical mechanics," Am. J. Phys. 48, 1074 (1980).

10. H. Butterfield, The Origins of Modern Science (Macmillan, New York, 1957)

11. I. B. Cohen, The Birth of a New Physics (Norton, New York, 1985), revised. 
12. A. C. Crombie, Medieval and Early Modern Science (Harvard U.P., Cambridge, MA, 1963), Vol.II.

13. E. J. Dijksetrhuis, The Mechanization of the World Picture (Clarendon, Oxford, 1964).

14. M. Jammer, Concepts of Force. A Study in the Foundations of Dynamics (Harvard U.P., Cambridge, MA, 1957).

15. Reference 12, p. 67.

16. Reference 12, p. 73.

17. D. P. Maloney, "Rule-governed approaches to physics. Newton's Third Law," Phys. Ed. 19, 37 (1984). 